\title{
Advantages of the Broadening Function (BF) over the Cross-Correlation Function (CCF)
}

\author{
Slavek M. Rucinski \\ David Dunlap Observatory, University of Toronto, P.O.Box 360, \\ Richmond Hill, Ontario, Canada L4C $4 Y 6$
}

\begin{abstract}
Performance of the cross-correlation function (CCF) is compared with the linear, algebraic equations, least-squares deconvolution directly giving the broadening function (BF). The many disadvantages of the CCF do not outweigh its simplicity so that the BF approach is strongly advocated.
\end{abstract}

\section{Disadvantages of the Cross-Correlation Function}

The cross-correlation function (CCF) is sometimes applied in estimates of the amount of the broadening of the spectral lines (e.g. for rotating stars, $v \sin i$ ) or for radial velocity measurements of multiple or binary objects. Such applications may result in systematic errors for the following reasons:

1. The CCF combines the broadening of the program spectrum with that of the template, with the resulting loss of resolution.

2. Outside of the main peak, which is normally used for radial velocity determination, the $\mathrm{CCF}$ is negative with a superimposed fringing pattern.

3. The above problem leads to difficulties with the definition of the baseline. This problem is particularly severe for sparse spectra which give stronger fringing patterns, because with fewer spectral lines, the positive fringes are usually stronger.

4. All of the above result in the shape of the CCF outside of the main correlation peak being dependent on the stellar spectrum. For the same star, different parts of the spectrum will define different CCF's.

\section{The Broadening Function}

The broadening function (BF), $B$ in $P=S * B$, where $P$ is the "program" (broadened) spectrum and $S$ is the "sharp" or the template spectrum, can be straightforwardly determined by solving algebraic, over-determined equations representing the above convolution. $S \& P$ are usually defined over thousands of pixels, while $B$ is usually defined over hundreds of pixels, so the equations can be cast as a standard least-squares problem with an over-determinacy typically of 10 -times or so.

The convolution operation which maps $S$ into a broad and/or binary-star spectrum $P, P\left(\lambda^{\prime}\right)=\int B\left(\lambda^{\prime}-\lambda\right) S(\lambda) d \lambda$, can be written as a set of algebraic 
linear equations, $\vec{P}=\mathbf{D} \vec{B}$. The array $\mathbf{D}(\vec{S})$ is created from the sharp-line spectrum vector $\vec{S}(n)$ by placing it as columns of $\mathbf{D}$ after shifting it downward by one index for each successive column. The BF is represented by a vector of the unknowns in the solution, $\vec{B}(m)$. The array $\mathbf{D}$ has the short dimension $m$ and the long dimension $n-m+1>m$; it accomplishes the mapping of $S \rightarrow P$.

Usually, a very good definition of $B$ results when some precautions are taken to take into account the linear dependencies caused by the continuum points and for the finite resolution which introduces coupling of the neighboring pixels in $B$. The reader is suggested to refer to Rucinski (1992, 1999, 2002). A description of the concept of the broadening functions with examples and detailed programming suggestions are available at the www site:

http://www.astro.utoronto.ca/ rucinski.

\section{Use of the BF's in the Binary Star Program at DDO}

We use the broadening functions to determine the radial velocities and rotational broadening of components in the short-period binary-star program at the David Dunlap Observatory. Until now, we have determined 75 radial velocity orbits. For references to the six papers with the first 60 orbits, see the interim paper Rucinski (2002).

The broadening function approach has permitted us to analyze close binaries in several multiple, visual/spectroscopic systems providing data which were too "difficult" before, with mixed sharp and rotationally broadened components. We found several bright $(<10 \mathrm{mag})$ triple systems where the spectra are dominated by the third, slowly rotating component which - although the fainter one in the visual system - defines the overall spectral appearance and produces sharp, easily identifiable spectral lines. The fact that such stars were not recognized could be explained by two non-exclusive causes: (1) The spectra were too complex to be handled by the CCF approach and such stars were previously abandoned; (2) Most of these spectra look like those of a single, slowly-rotating star and - paradoxically - the spectrum of the brighter close-binary component is not normally visible. The presence of the binary, which produces the broad spectral signature, manifests itself spectrally only through merging and blending of the more common, weaker lines and the general lowering of the continuum. The photometric variability signal of the brighter component is usually strongly "diluted" in the combined light of the system. We have became aware of those triple systems only thanks to detection of the low-amplitude photometric variability by the Hipparcos mission.

\section{References}

Rucinski, S. M. 1992, AJ 104, 1968

Rucinski, S. M. 1999, in: J. B. Hearnshaw \& C. D. Scarfe (eds.), Precise Stellar Radial Velocities, ASP Conf. Ser. Vol.185, p.82

Rucinski, S. M. 2002, AJ 124, 1746 